\title{
Cervical Cancer Screening Rates Among Chinese Women - China, 2015
}

\author{
Mei Zhang, ${ }^{1, x}$; Yijing Zhong, ${ }^{2, x}$; Zhenping Zhao'; Zhengjing Huang'; Xiao Zhang'; Chun Li'; \\ Maigeng Zhou ${ }^{1}$; Jing Wu ${ }^{1}$; Limin Wang ${ }^{1, * t}$; Xiaoying Zheng ${ }^{2, * *}$; Linhong Wang ${ }^{1, *}$
}

\section{Summary \\ What is already known about this topic? \\ Cervical cancer is the fourth most common cancer of women around the world. Age-adjusted incidence and mortality rates of cervical cancer were 11.78 and 3.29 per 100,000, respectively, in China in 2015. \\ What is added by this report? \\ Cervical cancer screening rates were $25.7 \%$ for women aged 20-64 years old and $31.4 \%$ for women aged 35-64 years old in China in 2015. Screening rates were lower in rural areas than in urban areas and varied across provinces. \\ What are the implications for public health practice? \\ Efforts should be made to continue to strengthen national and local policy initiatives, financial support, health education, and accessibility to women in rural areas for cervical cancer screening coverage.}

Cervical cancer is the fourth most common cancer of women around the world, and the only cancer deemed preventable and treatable with effective screening. Cervical cancer screening rates in China reached $20.7 \%$ for women aged 18 years old and above in 2010 and $21.4 \%$ for women aged 21 years old and above in 2013 (1-2). It is unknown whether cervical cancer screening rates improved in China after the country's serious efforts to strengthen screening coverage over the past decade. In order to understand the latest cervical cancer screening coverage in China, data from a nationally and provincially representative cross-sectional survey were analyzed, and screening rates were estimated based on answers to questionnaire interviews. Key demographic and geographic factors were analyzed via Rao Scott chi-square tests and logistic regression models. This study reported that cervical cancer screening rates improved from the past but remained at low levels among women in China. Efforts should be made to strengthen national and local policy initiatives and financial support on cervical cancer screening, carry out health education for women, and strengthen the accessibility of screening services to women in rural areas or areas with limited health-related resources.

According to the latest estimates, in 2018 there were 570,000 new cases and 311,000 new deaths of cervical cancer around the world, and without intervention the death tolls could increase $47.6 \%$ by 2040 (3). Cervical cancer is deemed preventable and treatable with mature prevention and control measures. The World Health Organization (WHO) was expected to adopt the draft entitled "Global Strategy Towards the Elimination of Cervical Cancer as a Public Health Problem" in 2020, which proposed cervical cancer intervention goals by 2030 such as $70 \%$ of women being screened with a high-precision test between 35-45 years of age (4). In China, cervical cancer had an age-adjusted incidence rate of 11.78 per 100,000 and a mortality rate of 3.29 per 100,000 in 2015 , ranking the sixth and eighth respectively among all cancers in women (5). While numerous measures including policy initiatives, financial support, and health education were taken to improve cervical cancer screening in China over the past decade, little is known about the latest screening coverage in China, especially in rural areas.

The latest cervical cancer screening rates were estimated in this study. In 2015, a cross-sectional survey was conducted in 298 districts/counties across 31 provincial-level administration divisions (PLADs), which were selected randomly from over 2,400 districts/counties to be representative of the whole population in the mainland of China as well as the population in every PLAD and specific regions, including urban and rural areas. The respondents aged 18 years old and above within every district/county were selected using a multi-stage, cluster-randomized sampling method (G). A set of questionnaires was used to collect data on chronic diseases and related behaviors by trained professionals from the local CDCs in China ( 7 ). In order to understand cervical cancer 
screening coverage, all female participants were asked whether they have ever had cervical cancer screening and the time of the most recent screening.

In 2015, of 88,250 households sampled, 100,543 female participants completed the survey from August to December, which yielded a $95.4 \%$ response rate. After excluding 18,948 female participants who were less than 20 years or over 64 years of age, 5,205 participants who were unclear whether they have been screened, 586 participants who did not respond to the cervical cancer screening question, and 517 participants who had incomplete sociodemographic data, a final sample of 75,287 women aged 20 to 64 years old was analyzed in this study. The study was approved by the Ethical Committee of the National Centre for Chronic and Non-communicable Disease Control and Prevention, China CDC. All participants provided written informed consent.

Weighting was applied to all statistical analyses for both national and region-specific estimates (8). The weighted proportion for the characteristics of Chinese women aged 20 to 64 years old was reported. Chisquare tests were used for comparisons between unordered categorical variables, whereas logistic regression models were used to examine the trend for ordered categorical variables. Taylor linearization methods with a finite population correction were used to estimate standard errors (SE) accounting for the complex sampling design. Statistical significance was determined as a two-sided $p<0.05$. All statistical analyses used software SAS (version 9.4, SAS Institute Inc., Cary, USA).

According to the weighted proportions, $41.7 \%$ of women aged 20 to 64 years old were from urban areas and $42.4 \%$ from China's eastern region. (Table 1) A total of 5,205 participants who were unclear about their screening history were excluded from the final analysis and constituted about $7 \%$ of the final sample. These participants had similar age and health insurance status but were slightly more rural, less educated, and had lower likelihood of health examination in the past three years than the final sample.

Overall, cervical cancer ever screening rate was $25.7 \%$ in women aged $20-64$ years old and $31.4 \%$ in women aged 35-64 years old. The urban screening rate was significantly higher than rural screening rate (30.0\% vs. $22.6 \%, p<0.001$ ). The $35-44$ age group had significantly higher screening rate $(36.9 \%)$ than other age groups $(p<0.001)$. The eastern region showed significantly higher screening rate $(33.7 \%)$ than other regions only for women in urban areas $(p<0.001)$, whereas there was no statistically significant difference in screening rate across the geographical regions for women in rural areas. (Table 2)

Moreover, among the women who were screened, about $90 \%$ of them were screened within the past three years.

Women with college or above education and with high school education had significantly higher screening rates $(30.8 \%$ and $30.5 \%$, respectively) than women with middle school education $(25.6 \%)$ and with primary or less education $(21.2 \%, p<0.001)$. Although about one-fifth of participants did not reveal their household income, available data showed that screening increased with advancing income $(p<0.001)$.

Retired women had significantly higher screening rates $(34.4 \%)$ than employed women $(27.2 \%)$, women doing housework $(21.4 \%)$, and unemployed women $(17.2 \%, p<0.001)$. Women without health insurance had significantly lower screening than women with health insurance $(12.9 \%$ vs. $26.2 \%, p<0.001)$. Women without a health examination in the past three years had significantly lower screening rate than women with health examinations in the past three years $(16.6 \% \mathrm{vs}$. $40.0 \%, p<0.001)$. Women with self-assessed poor or fair health status had significantly higher screening rate than women with self-assessed good health status (27.9\% vs. $23.1 \%, p<0.001)$.

Provincial-level data further showed that screening rates varied widely across the 31 PLADs. The screening rates in 5 provinces, including Beijing and Shanghai, exceeded $35 \%$, whereas three provinces, Tibet, Anhui, and Hebei, had screening rates of less than $15 \%$. (Figure 1)

\section{DISCUSSION}

This study showed that cervical cancer screening rates remained low in China, especially for women in rural areas. In 2015, $25.7 \%$ of women aged 20-64 years old and $31.4 \%$ of women aged $35-64$ years old ever had cervical cancer screening. About $90 \%$ of screened women were screened within the past 3 years. Efforts should be made to continue to strengthen national and local policy initiatives, financial support, health education, and accessibility for women in rural areas for cervical cancer screening coverage. Cervical cancer is preventable and treatable, and increasing cervical cancer screening is critical to allow for early detection of precancerous lesions and protect women against cervical cancer.

Gaps still exist in cervical cancer screening coverage 
TABLE 1. Sociodemographic characteristics of female participants aged 20-64 years old of China Chronic Disease and Nutrition Surveillance, 2015.

\begin{tabular}{|c|c|c|}
\hline Characteristics & No. of participants $(n=75,287)$ & Weighted Proportion $(\%)^{*}$ \\
\hline \multicolumn{3}{|l|}{ Age (years old) } \\
\hline $20-24$ & 2,707 & 15.3 \\
\hline $25-34$ & 11,251 & 22.2 \\
\hline $35-44$ & 15,107 & 26.9 \\
\hline $45-54$ & 24,392 & 20.3 \\
\hline $55-64$ & 21,830 & 15.4 \\
\hline \multicolumn{3}{|l|}{ Residence } \\
\hline Urban & 32,639 & 41.7 \\
\hline Rural & 42,648 & 58.3 \\
\hline \multicolumn{3}{|l|}{ Location } \\
\hline East & 28,365 & 42.4 \\
\hline Middle & 21,649 & 32.3 \\
\hline West & 25,273 & 25.4 \\
\hline \multicolumn{3}{|l|}{ Education } \\
\hline Primary or less & 36,781 & 35.3 \\
\hline Secondary & 22,500 & 32.5 \\
\hline High & 9,444 & 15.6 \\
\hline College or above & 6,562 & 16.6 \\
\hline \multicolumn{3}{|l|}{ Household income per capita (CNY) } \\
\hline Q1 $(<6,000)$ & 14,503 & 15.6 \\
\hline Q2 $(6,000-11,999)$ & 15,543 & 19.7 \\
\hline Q3 $(12,000-21,600)$ & 17,165 & 23.0 \\
\hline Q4 $(21,600$ and above $)$ & 15,745 & 24.6 \\
\hline Don't know/refused to answer & 12,331 & 17.1 \\
\hline \multicolumn{3}{|l|}{ Employment status } \\
\hline Employed & 51,746 & 69.8 \\
\hline Housework & 14,694 & 18.4 \\
\hline Retired & 5,325 & 4.2 \\
\hline Unemployed & 3,522 & 7.6 \\
\hline \multicolumn{3}{|l|}{ Health insurance coverage } \\
\hline No & 2,194 & 4.3 \\
\hline Yes & 73,093 & 95.7 \\
\hline \multicolumn{3}{|l|}{ Health examination in the past 3 years } \\
\hline No & 46,702 & 53.6 \\
\hline Yes & 28,585 & 46.4 \\
\hline \multicolumn{3}{|l|}{ Self-assessed health status } \\
\hline Poor or fair & 43,475 & 61.1 \\
\hline Good & 31,812 & 38.9 \\
\hline
\end{tabular}

* Proportions are weighted to represent the national total population with poststratification for age, gender, and urban/rural residence.

in China with $36.9 \%$ screening rates in China compared to the $70 \%$ WHO goal for the $35-44$ age group, which was mainly due to the absence of an organized, population-based cervical cancer screening system in China. However, screening rates in China showed significant improvement from the past. 
TABLE 2. Cervical cancer screening rates among Chinese women aged 20-64 years old by sociodemographic factors China, 2015*.

\begin{tabular}{|c|c|c|c|c|}
\hline Item & Total $(\%)(95 \% \mathrm{Cl})$ & Urban $(\%)(95 \% \mathrm{Cl})$ & Rural (\%)(95\%Cl) & $p$-value \\
\hline Total & $25.7(24.1-27.2)$ & $30.0(27.7-32.2)$ & $22.6(20.3-24.9)$ & $<0.001$ \\
\hline \multicolumn{5}{|l|}{ Age (years old) } \\
\hline $20-24$ & $7.5(6.1-8.9)$ & $7.0(4.7-9.3)$ & $7.9(6.2-9.6)$ & 0.569 \\
\hline $25-34$ & $22.1(20.1-24.0)$ & $26.6(23.6-29.5)$ & $18.3(15.5-21.2)$ & $<0.001$ \\
\hline $35-44$ & $36.9(34.5-39.4)$ & $43.6(39.4-47.8)$ & $32.2(29.4-35.1)$ & $<0.001$ \\
\hline $45-54$ & $32.4(30.4-34.5)$ & $38.3(35.2-41.4)$ & $28.8(25.8-31.9)$ & $<0.001$ \\
\hline $55-64$ & $20.2(18.6-21.9)$ & $25.1(22.5-27.6)$ & $17.1(14.7-19.5)$ & $<0.001$ \\
\hline$p$-value for trend & $<0.001$ & $<0.001$ & $<0.001$ & \\
\hline \multicolumn{5}{|l|}{ Geographic location } \\
\hline East & $29.1(27.0-31.3)$ & $33.7(30.1-37.2)$ & $24.4(21.1-27.7)$ & 0.001 \\
\hline Middle & $22.3(19.0-25.6)$ & $26.9(22.9-31.0)$ & $20.3(15.9-24.7)$ & 0.041 \\
\hline West & $24.1(21.9-26.4)$ & $25.0(21.8-28.3)$ & $23.6(20.3-26.9)$ & 0.582 \\
\hline$p$-value for difference & $<0.001$ & 0.001 & 0.275 & \\
\hline \multicolumn{5}{|l|}{ Education } \\
\hline Primary or less & $21.2(19.2-23.1)$ & $22.0(18.9-25.2)$ & $20.9(18.4-23.4)$ & 0.607 \\
\hline Middle & $25.6(23.5-27.7)$ & $30.2(26.9-33.4)$ & $23.2(20.3-26.1)$ & 0.005 \\
\hline High & $30.5(28.3-32.7)$ & $33.0(30.1-36.0)$ & $26.9(23.1-30.7)$ & 0.024 \\
\hline College or above & $30.8(27.9-33.7)$ & $32.6(29.1-36.1)$ & $24.3(19.5-29.1)$ & 0.013 \\
\hline$p$-value for trend & $<0.001$ & $<0.001$ & 0.013 & \\
\hline \multicolumn{5}{|c|}{ Household income per capita (CNY) } \\
\hline Q1 $(<6,000)$ & $19.4(17.4-21.4)$ & $19.2(16.6-21.8)$ & $19.4(17.0-21.9)$ & 0.913 \\
\hline Q2 $(6,000-11,999)$ & $22.0(20.3-23.8)$ & $24.6(21.5-27.7)$ & $21.0(18.8-23.2)$ & 0.072 \\
\hline Q3 $(12,000-21,599)$ & $27.8(25.9-29.7)$ & $29.6(27.1-32.1)$ & $26.3(23.2-29.4)$ & 0.142 \\
\hline Q4 $(21,600$ and above $)$ & $33.7(31.4-35.9)$ & $35.3(32.4-38.3)$ & $30.4(26.7-34.2)$ & 0.074 \\
\hline Don't know/refused to answer & $21.2(18.7-23.8)$ & $27.2(22.8-31.6)$ & $17.9(15.0-20.8)$ & $<0.001$ \\
\hline$p$-value for trend ${ }^{\dagger}$ & $<0.001$ & $<0.001$ & $<0.001$ & \\
\hline \multicolumn{5}{|l|}{ Employment status } \\
\hline Employed & $27.2(25.7-28.7)$ & $31.7(29.1-34.3)$ & $24.1(22.0-26.2)$ & $<0.001$ \\
\hline Housework & $21.4(18.5-24.3)$ & $25.0(20.8-29.1)$ & $19.8(15.9-23.7)$ & 0.105 \\
\hline Retired & $34.4(31.4-37.3)$ & $34.8(31.5-38.0)$ & $31.5(24.0-39.0)$ & 0.469 \\
\hline Unemployed & $17.2(14.8-19.6)$ & $19.9(17.6-22.2)$ & $14.4(10.1-18.6)$ & 0.048 \\
\hline$p$-value for difference & $<0.001$ & $<0.001$ & $<0.001$ & \\
\hline \multicolumn{5}{|l|}{ Health insurance coverage } \\
\hline No & $12.9(10.4-15.4)$ & $11.5(8.8-14.2)$ & $15.5(10.3-20.7)$ & 0.181 \\
\hline Yes & $26.2(24.7-27.8)$ & $31.3(28.9-33.6)$ & $22.8(20.5-25.1)$ & $<0.001$ \\
\hline$p$-value for difference & $<0.001$ & $<0.001$ & 0.021 & \\
\hline \multicolumn{5}{|c|}{ Health examination in the past 3 years } \\
\hline No & 16.6(15.3-17.9) & $16.5(14.8,18.2)$ & $16.6(14.8-18.4)$ & 0.95 \\
\hline Yes & $40.0(37.9-42.1)$ & $42.3(39.6,44.9)$ & $37.1(33.7-40.5)$ & 0.029 \\
\hline$p$-value for difference & $<0.001$ & $<0.001$ & $<0.001$ & \\
\hline \multicolumn{5}{|l|}{ Self-assessed health status } \\
\hline Poor or fair & $27.9(26.2-29.5)$ & $32.5(30.3-34.7)$ & $24.6(22.2-27.1)$ & $<0.001$ \\
\hline Good & $23.1(21.5-24.8)$ & $27.1(24.4-29.8)$ & $20.2(17.8-22.5)$ & $<0.001$ \\
\hline$p$-value for difference & $<0.001$ & $<0.001$ & $<0.001$ & \\
\hline
\end{tabular}

* Screening rates are all weighted proportions.

† Participants answering "don't know/refused to answer" were not included in the trend test. 




FIGURE 1. Cervical cancer screening rates among Chinese women at the provincial level in China in 2015.

Compared to the current screening rates of $25.7 \%$ for women aged 20 to 64 years old, screening rates were only $20.7 \%$ for women aged 18 years old and above in 2010 and $21.4 \%$ for women aged 21 years old and above in 2013. In particular, screening rates for the $35-64$ age group increased remarkably from $26.7 \%$ in 2013 to $31.4 \%$ in 2015 , which was the target age group of China's cervical cancer screening program in rural areas launched in 2009 (9). Rural screening also increased remarkably from $16.9 \%$ in 2010 to $22.6 \%$ in 2015 , and rural screening coverage in the western region notably caught up with rural screening coverage in the middle and eastern regions. Furthermore, over $90 \%$ of screening took place within the past 3 years among women who ever had the screening, which indicated that most women followed the guidelines for screening every 3 years. The main reason may be attributable to China's national policy efforts in increasing cervical cancer screening coverage. For example, China's Women Development Program (2011-2020) strived for $80 \%$ screening coverage of women's common diseases. The National Screening Program for Cervical Cancer and Breast Cancer in Rural Areas provided free cervical cancer screening to about 10 million women aged 35-64 years old in rural areas annually since 2009 , constituting about $20 \%$ of all women aged 35-64 years old in rural China in 2012-2014 (10). Efforts were also made to strengthen the screening system and technical personnel training, especially training of screening techniques of local staff. In addition, the study reveals significant variances in screening coverage across PLADs, indicating that different policies and strategies may be at work on the ground. Given the positive improvement in cervical cancer screening rates, the central and local governments should continue to strengthen policy and financial support as well as health education to women in order to increase cervical cancer screening coverage in China.

This study also found that women in rural areas, as well as women who were unemployed, who did not have health insurance, and who did not have a health examination in the past three years had significantly lower cervical cancer screening rates. These suggest that with limited resources, targeting women of these subgroups should be a priority.

This study is limited by response bias as $7 \%$ of the initial respondents that were unclear of their screening history were excluded from the final analysis, and those excluded were slightly more rural, were less educated, and had less health examination than the final sample. Assuming these respondents were all unscreened would lower final screening rates by about $1 \%-2 \%$, though this would not change the conclusion. Misclassification may also occur as the respondents might incorrectly recall their screening history. Furthermore, the questionnaire did not ask for the reason of not having had cervical cancer screening, which thus fails to offer 
an additional opportunity to examine the factors affecting screening accessibility.

In conclusion, this study provides a 2015 updated assessment of the largest nationwide and populationbased self-reported history of cervical cancer screening in China. About one-quarter of Chinese women ever had cervical cancer screening. Efforts should be made to continue to strengthen national and local policy initiatives and financially support cervical cancer screening coverage, enhance health education, and improve the accessibility of screening services to women in rural areas, without employment, without health insurance coverage, and without health examination in the past three years.

doi: $10.46234 / \mathrm{ccdcw} 2020.128$

* Corresponding authors: Linhong Wang, wanglinhong@ncncd. chinacdc.cn; Xiaoying Zheng, xzheng@pku.edu.cn; Limin Wang, wanglimin@ncncd.chinacdc.cn.

\footnotetext{
National Center for Chronic and Non-Communicable Disease Control and Prevention, Chinese Center for Disease Control and Prevention, Beijing, China; ${ }^{2}$ Institute of Population Research, Peking University, Beijing, China.

\& Joint first authors.
}

Submitted: March 25, 2020; Accepted: June 04, 2020

\section{REFERENCES}

1. Wang BH, He MF, Chao A, Engelgau MM, Saraiya M, Wang LM, et al. Cervical cancer screening among adult women in China, 2010. Oncologist 2015;20(6):627 - 34. http://dx.doi.org/10.1634/theonco logist.2014-0303.
2. Bao HL, Zhang L, Wang LM, Zhang M, Zhao ZP, Fang LW, et al. Significant variations in the cervical cancer screening rate in China by individual-level and geographical measures of socioeconomic status: a multilevel model analysis of a nationally representative survey dataset. Cancer Med 2018;7(5):2089 - 100. http://dx.doi.org/10.1002/cam4. 1321.

3. International Agency for Research on Cancer. Cervix uteri source: globocan 2018. https://gco.iarc.fr/today/data/factsheets/cancers/23Cervix-uteri-fact-sheet.pdf. [2020-01-04].

4. World Health Organization. Draft: global strategy towards eliminating cervical cancer as a public health problem. https://www.who.int/docs/ default-source/cervical-cancer/cerv-cancer-elimn-strategy-16dec12pm.pdf. [2020-01-04].

5. Zheng RS, Sun KX, Zhang SW, Zeng HM, Zou XN, Chen R, et al. Report of cancer epidemiology in China, 2015. Chin J Oncol 2019;41(1):19 - 28. http://dx.doi.org/10.3760/cma.j.issn.0253-3766. 2019.01.005. (In Chinese).

6. Li YC, Wang LM, Jiang Y, Zhang M, Wang LH. Risk factors for noncommunicable chronic diseases in women in China: surveillance efforts. Bull World Health Organ 2013;91(9):650 - 60. http://dx.doi.org/ 10.2471/BLT.13.117549.

7. Guan YQ, Zhang M, Zhang X, Zhao ZP, Huang ZJ, Li C, et al. Hypertension prevalence, awareness, treatment, control, and associated factors in the labor force population - China, 2015. China CDC Weekly 2020;2(10):147-55. http://weekly.chinacdc.cn/en/article/doi/ $10.46234 / \mathrm{ccdcw} 2020.040$

8. Hu N, Jiang Y, Li YC, Chen YD. Data weighting methods for China Chronic Disease Surveillance (2010). Chin J Health Stat 2012;29(3): 424-6. http://www.wanfangdata.com.cn/details/detail.do?_type=perio\& $\mathrm{id}=$ zgwstj201203045. (In Chinese).

9. Bao HL, Wang LH, Wang LM, Fang LW, Zhang M, Zhao ZP, et al. Study on the coverage of cervical and breast cancer screening among women aged 35-69 years and related impact of socioeconomic factors in China, 2013. Chin J Epidemiol 2018;39(2):208 - 12. http://dx.doi.org/ 10.3760/cma.j.issn.0254-6450.2018.02.014. (In Chinese).

10. Di JL. Opportunities and challenges in cervical cancer screening program in China: SWOT analysis for implementation of program. Qual Prim Care 2017;25(5):282-8. https://primarycare.imedpub.com/ opportunities-and-challenges-in-cervical-cancerscreening-program-inchina-swot-analysis-forimplementation-of-program.php?aid=20761. 